Check for updates

Cite this: RSC Adv., 2019, 9, 9755

\title{
Enhanced, parallel liquid crystal alignment based on polystyrene substituted with phthalimidoyl groups
}

\begin{abstract}
Taehyung Kim, Changha Ju, Chanhyuk Park and Hyo Kang (DD*
A series of polystyrene (PS) polymers substituted with phthalimide (PPH\#), where \# is the degree of substitution, were synthesized via a polymer analogous reaction to investigate liquid crystal (LC) alignment behaviors of LC cells fabricated using PPH\# films. The PPH\# films exhibited higher optical transparency in the visible light region compared to a commercial polyimide (PI) film. The transmittance of each PPH\# film ranged from 91 to 93\%, whereas that of PI was $80.5 \%$ at $550 \mathrm{~nm}$. The LC cell fabricated with a pristine PS film showed planar LC alignment perpendicular with respect to the rubbing direction. The introduction of the phthalimide group to the side chain of PS diverted the LC alignment direction from perpendicular to parallel with respect to the rubbing direction. The LC cells fabricated with PPH\# films exhibited planar LC alignment parallel with respect to the rubbing direction when the degree of substitution of phthalimidoyl moieties was $>20$ mol\%. In addition, the long-term stability of the LC cell composed of pristine PS was very low. However, the long-term stability of the LC cells fabricated with the PPH\# series was remarkably enhanced in addition to their ultraviolet stability. Therefore, this study contributes to the development of planar and parallel LC alignments on PS derivatives and can be used in the next-generation display industry for the production of flexible displays.
\end{abstract}

\author{
Received 13th February 2019 \\ Accepted 21st March 2019 \\ DOI: 10.1039/c9ra01131e \\ rsc.li/rsc-advances
}

\section{Introduction}

Liquid crystals (LCs), the so-called mesogens, are materials that exhibit fluidity similar to a conventional liquid but with some degree of ordering under certain conditions. Typically, LCs exhibit anisotropic physicochemical properties due to their distinct chemical structure, which can be caused by temperature and solution concentration changes. ${ }^{1}$ Among the various types of LCs, thermotropic LCs adopt an isotropic liquid phase above a critical temperature. When cooled below the critical temperature, they convert from liquid to the mesophase as the LC molecular order changes. ${ }^{2}$ To manifest ordered properties, mechanical rubbing using a polymeric alignment layer and photoalignment of LC molecules by irradiation of polarized light onto a photoreactive substance have been widely utilized. ${ }^{3}$

Recently, reactive mesogens (RMs) containing reactive groups as well as core, linkage, terminal, and lateral groups have been widely studied. ${ }^{4-7}$ The chemical reaction between RM reactive groups solidifies the LC materials to form a polymer, which can be used in many diverse films and devices for various applications. RMs have been widely used for non-display application and as compensation films and micro-lenses in various displays. RMs in non-display applications are used for

Department of Chemical Engineering, Dong-A University, 37 Nakdong-Daero 550beon-gil, Saha-gu, Busan 49315, Republic of Korea.E-mail: hkang@dau.ac.kr; Fax: +82 51200 7728; Tel: +8251200 7720 physicochemical reactions or as mechanically transformable or stimulus-sensitive components, e.g., actuator, in controlling systems. Developments in RM technology have focused on shape and volumetric changes in various systems. RMs have been applied in diverse fields such as soft biology, biomedicine, and microfluidic biochips. ${ }^{8-11}$ Likewise, RMs are attractive materials that can be adapted for use in various fields by controlling the physicochemical properties of actuators via environmental changes.

More recently, LCs have been extensively adopted as a demand for foldable and stretchable displays has increased rapidly. LC molecules that exhibit optical and dielectric anisotropy in one direction on the alignment layer have been widely used in displays after alignment. Most LC aligning behaviors can be triggered by physicochemical interaction with aligned polymeric layers. Polymeric thin films have been applied extensively for LC flat-panel displays to induce unidirectional LC alignment. In particular, polyimide (PI) has been applied in LC aligning films and is widely used in the display industry. Rubbed PI films generally align the LC molecules parallel with respect to the rubbing direction. The mechanisms underlying these phenomena have been previously described as physicochemical interactions between the polymeric films and LC molecules and microgroove effects of rubbed films. ${ }^{12-14}$ Although PI has been extensively used in the display industry, PI precursors must be cured at high temperature to obtain the final PI film and the film itself exhibits an intrinsically yellowish 
color. ${ }^{15,16}$ Polystyrene (PS) films that induce the homogeneous alignment of LC molecules perpendicular with respect to the rubbing direction can be produced at low temperature and exhibit high transmittance in the visible wavelength range, which is suitable for flexible displays. ${ }^{17-19}$ For PS films generating weak azimuthal anchoring energy, the long-term stability of LC cells made with PS films is insufficient for the display industry. ${ }^{20,21}$ To overcome this issue, a phytochemical material, coumarin, was introduced to the LC alignment to enhance the stability of the perpendicular LC alignment while maintaining the alignment by applying a coumarin-moiety-containing PS as a LC alignment film. ${ }^{22}$ Unfortunately, few studies have demonstrated enhanced LC orientation performance parallel with respect to the rubbing direction by introducing specific moieties into the PS side chains.

In this study, PS substituted with phthalimide (PPH\#) was synthesized by introducing isoindoline-1,3-dione, a representative moiety in heterocyclic PI, to the polymer side chain to divert the LC alignment direction from perpendicular to parallel with respect to the rubbing direction. We examined the enhanced parallel LC alignment performance and degree of substitution effect of the phthalimide side group in the polymer chain on the LC alignment properties. The optical and surface properties of the LC cells composed of $\mathrm{PPH}$ \# polymer films were also characterized.

\section{Experimental}

\subsection{Materials}

4-Chloromethylstyrene, phthalimide potassium salt, and $4^{\prime}$ pentyl-4-biphenylcarbonitrile $\left(5 \mathrm{CB}, n_{\mathrm{e}}=1.7360, n_{\mathrm{o}}=1.5442\right.$, and $\Delta \varepsilon=14.5$, where $n_{\mathrm{e}}, n_{\mathrm{o}}$, and $\Delta \varepsilon$ represent the extraordinary refractive indexes, ordinary refractive indexes, and dielectric anisotropy, respectively) were purchased from Aldrich Chemical Co. Polystyrene (PS) (INEOS Styrolution Group GmbH, GPPS $147 \mathrm{~F}$ ) (average $M_{\mathrm{w}} ; 35000 \mathrm{~g} \mathrm{~mol}^{-1}$, softening point range; 123$128{ }^{\circ} \mathrm{C}$ ) and polyimide (PI) alignment agents (Nissan Chemical Industries Ltd., SE-7492K) were used as-received. $N, N^{\prime}$-Dimethylformamide and methanol were dried over a molecular sieve (4 $\AA$ ). Tetrahydrofuran (THF) was dried by refluxing with benzophenone and sodium followed by distillation. 4-Chloromethylstyrene was purified using column chromatography on silica gel with hexane as an eluent to remove impurities and inhibitors (tert-butylcatechol and nitroparaffin). Poly(4chloromethylstyrene) (PPH0) was synthesized via analogous free radical polymerization of 4-chloromethylstyrene using 2,2' azobisisobutyronitrile (AIBN) under a nitrogen atmosphere. AIBN (Junsei Chemical Co., Ltd.) was used as an initiator and was purified by crystallization from methanol. All other reagents and solvents were used as-received.

\subsection{Preparation of the PS substituted with phthalimidoyl group (PPH\#)}

The following procedure was used to synthesize a series of PS substituted with phthalimide, $\mathrm{PPH}$, where \# signifies the molar ratio of the phthalimide moiety in the polymer side chain. The synthesis of PS modified with phthalimide homopolymer (PPH100) will be provided as an example. A mixture of phthalimide potassium salt $(1.82 \mathrm{~g}, 9.84 \mathrm{mmol}, 150 \mathrm{~mol} \%$ compared with PPH0) in $N, N^{\prime}$-dimethylformamide (DMF, 30 $\mathrm{mL}$ ) was heated to $70{ }^{\circ} \mathrm{C}$. A PPH0 ( $\left.1 \mathrm{~g}, 6.56 \mathrm{mmol}\right)$ solution in DMF $(20 \mathrm{~mL})$ was added to the mixture and stirred magnetically at $70{ }^{\circ} \mathrm{C}$ for $6 \mathrm{~h}$ under a nitrogen atmosphere. The solution mixture was cooled to room temperature and poured into methanol to obtain a white precipitate. The precipitate was further purified by several reprecipitation cycles from DMF solution into methanol and washed with hot methanol by Soxhlet extraction to remove any unreacted materials. The precipitate was obtained in yields of $>85 \%$ after drying in vacuo overnight. The degree of substitution of chloromethyl to phthalimidoyl methyl group was almost $100 \%$ within the experimental error. The proton nuclear magnetic resonance $\left({ }^{1} \mathrm{H}-\mathrm{NMR}\right)$ peaks for PPH100 were as follows;

${ }^{1} \mathrm{H}-\mathrm{NMR}$ of PPH100 [400 MHz, $\left.\mathrm{CDCl}_{3}, \delta / \mathrm{ppm}\right]: 0.9-2.0(\mathrm{~m}$, $3 \mathrm{H},\left(-\mathrm{CH}_{2}-\mathrm{CH}-\mathrm{Ph}-\right)$ ), 4.55-4.85 (s, 2H, (-Ph- $\left.\left.\mathrm{CH}_{2}-\mathrm{N}-\right)\right)$, 6.25-7.2 (d, $\left.4 \mathrm{H},\left(-\mathrm{CH}-\mathrm{Ph}-\mathrm{CH}_{2}-\right)\right), 7.45-7.9(\mathrm{~d}, 4 \mathrm{H},(-\mathrm{N}-(\mathrm{C}=\mathrm{O})-\mathrm{C}-\mathrm{CH}-))$.

Other polystyrene derivates, including phthalimidoyl side groups, were synthesized using the same procedure as PPH100, except for differing amounts of phthalimide potassium salt in the reaction. For instance, PPH80, PPH60, PPH40, and PPH20 were prepared using $0.97 \mathrm{~g}(5.25 \mathrm{mmol}), 0.73 \mathrm{~g}$ (3.94 $\mathrm{mmol})$, $0.49 \mathrm{~g}(2.63 \mathrm{mmol})$, and $0.25 \mathrm{~g}(1.31 \mathrm{mmol})$ of phthalimide potassium salt, respectively.

\subsection{Film preparation and LC cell assembly}

Solutions of PS and PPH\# in chloroform (1 wt\%) were prepared and a PI solution was applied as-received. The solutions were filtered using a polytetrafluoroethylene (PTFE) membrane with a pore size of $0.45 \mu \mathrm{m}$. Thin films of each polymer were prepared by spin-coating ( $2000 \mathrm{rpm}, 60 \mathrm{~s}$ ) onto glass substrates and silicon wafers. Additionally, PI alignment agents were spin coated (3000 rpm, $40 \mathrm{~s}$ ) onto the glass substrates. The PI films were prebaked at $80{ }^{\circ} \mathrm{C}$ for $15 \mathrm{~min}$ and fully baked at $220{ }^{\circ} \mathrm{C}$ for $45 \mathrm{~min}$. All polymer films were rubbed using a rubbing machine (Sciencetown, SRMS-50-M) with a rubbing number and depth of 1 and $0.3 \mathrm{~mm}$, respectively. The LC cells were constructed by assembling the films coated onto the glass substrates and $\mathrm{Si}$ wafers in an anti-parallel direction using spacers with a thickness of $4.25 \mu \mathrm{m}$. The cells were then filled with nematic LC, $4^{\prime}$ pentyl-4-biphenylcarbonitrile (5CB), using the capillary phenomenon, and were subsequently sealed with epoxy glue.

\subsection{Instrumentation}

The proton nuclear magnetic resonance $\left({ }^{1} \mathrm{H}-\mathrm{NMR}\right)$ spectroscopy was performed using a Bruker Agilent MR400 DD2 NMR spectrometer with a $400 \mathrm{MHz}$ magnet. The gel permeation chromatography (GPC) was used to measure the number average molecular weight $\left(M_{\mathrm{n}}\right)$ and polydispersity index $\left(M_{\mathrm{w}} / M_{\mathrm{n}}\right)$ of the synthesized polymer with respect to polystyrene standards using tetrahydrofuran (THF) as the eluent and an UV detector. The differential scanning calorimetry (DSC) thermogram was obtained using a differential scanning calorimeter (TA 
Instruments, Q-10). The optical transmittance of the polymer films on the glass substrates was acquired using an ultravioletvisible (UV-Vis) spectrophotometer (MECASYS Co. LTD., OPTIZEN POP). The polarized optical microscopy (POM) images of the LC cells were obtained using an optical microscope (OLYMPUS, BX51) equipped with a polarized and digital camera (Tucsen Photonics, ISH300). The Fourier-transform infrared (FT-IR) dichroic spectra and polar diagrams were obtained using a FT-IR spectrometer (Thermo Fisher Scientific, NICOLET 380) equipped an infrared polarizer composed of zinc selenide from 4000 to $400 \mathrm{~cm}^{-1}$ with a resolution of $4 \mathrm{~cm}^{-1}$. The surface morphology of the unrubbed and rubbed polymer films in an area of $5 \times 5 \mu \mathrm{m}^{2}$ was examined using atomic force microscopy (AFM) (Digital Instruments, MMAFM-2) in tapping mode (spring constant of the cantilever: $42 \mathrm{~N} \mathrm{~m}^{-1}$, scan rate: 0.986 $\mathrm{Hz})$.

\section{Result \& discussion}

\subsection{Synthesis and characterization of PS substituted with phthalimidoyl group (PPH\#)}

Fig. 1 shows the synthetic routes used to obtain PS substituted with phthalimide, $\mathrm{PPH}$ (\# = 20, 40, 60, 80, and 100), where \# signifies the molar ratio (\%) of phthalimidolyl side groups in the polymers. The different degrees of substitution (\%) in the copolymers such as PPH20, PPH40, PPH60, and PPH80 were obtained by changing the amounts of phthalimide potassium salt in the polymer analogous reaction. The degree of substitution from chloromethyl to phthalimidoyl methyl was approximately $100 \%$ when $150 \mathrm{~mol} \%$ of the phthalimide potassium salt was used at $70{ }^{\circ} \mathrm{C}$ for $6 \mathrm{~h}$, as determined by analyzing the respective proton peaks of styrene substituted with phthalimide homopolymer (PPH100). The chemical compositions of the monomeric units in the homopolymer of $\mathrm{PPH} \#$ series, PPH100, were confirmed by analyzing respective proton nuclear magnetic resonance $\left({ }^{1} \mathrm{H}-\mathrm{NMR}\right)$ peaks from the ${ }^{1} \mathrm{H}$-NMR spectrum in Fig. 2.

The ${ }^{1} \mathrm{H}$-NMR spectrum of PPH100 indicated the presence of protons from the methylstyrene moiety $(\delta / \mathrm{ppm}=0.90-2.00$ $(3 \mathrm{H}), 4.55-4.85(2 \mathrm{H}), 6.25-7.20(4 \mathrm{H})$, peaks a-c). From the pertinent spectrum, the proton peaks of the phthalimide side chains $(\delta / \mathrm{ppm}=7.45-7.9(4 \mathrm{H})$, peak $\mathrm{d})$ indicated inclusion of the phthalimide moiety in PPH100. Thus, the degree of

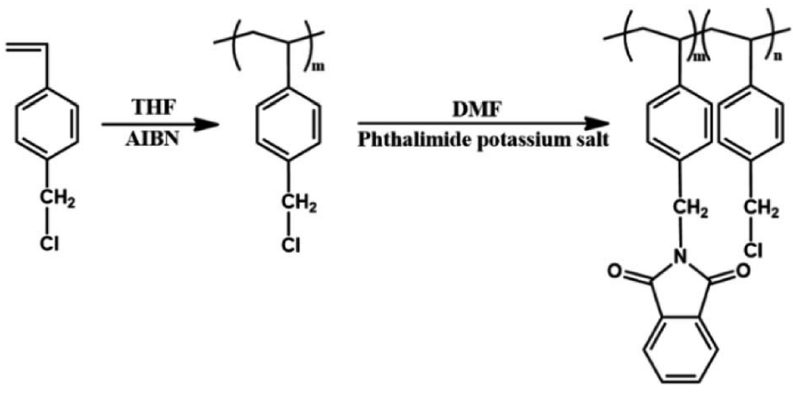

Fig. 1 Synthetic route for phthalimide modified polystyrene (PPH\#).

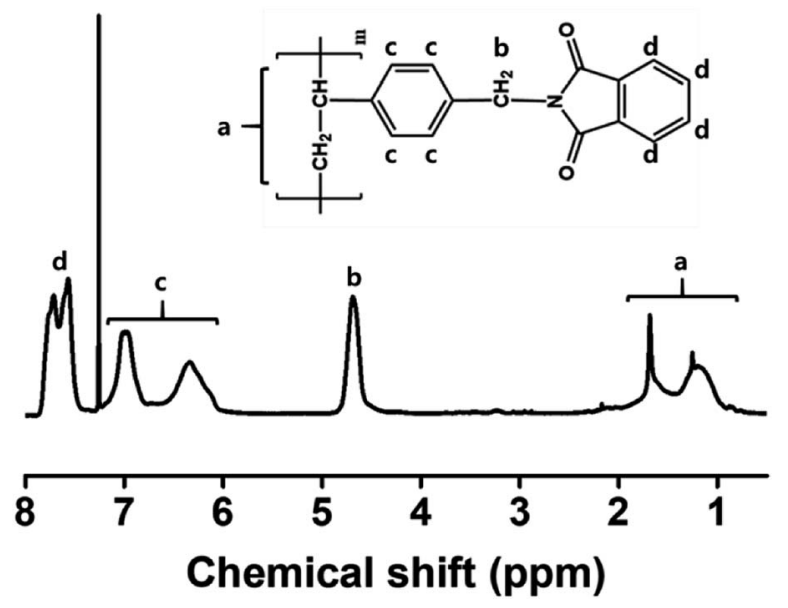

Fig. 2 Proton nuclear magnetic resonance $\left({ }^{1} \mathrm{H}-\mathrm{NMR}\right)$ spectrum of $\mathrm{PPH} 100$.

substitution from chloromethyl to phthalimidoyl methyl was approximately $100 \%$ when $150 \mathrm{~mol} \%$ of the phthalimide potassium salt was used under the specified reaction conditions. The phthalimide content was calculated by comparing the integrated area under the proton peaks of phthalimide $(\delta /$ ppm $=7.45-7.9$, peak d) and those corresponding to the chloromethyl side chains $(\delta / \mathrm{ppm}=4.55-4.85$, peak $\mathrm{b})$. The calculated phthalimide contents of the $\mathrm{PPH}$ series are shown in Table 1. Similar integrations and calculations were performed for the PPH20, PPH40, PPH60, and PPH80 samples, and were typically within $\pm 5 \%$ of the expected values. The average molecular weights $\left(M_{\mathrm{n}}\right)$ of these polymer series synthesized from the PPH0 $\left(M_{\mathrm{n}}=22000\right)$ were always larger than 24000 indicating that polymer modification from PPH0 to the polymers gave rise to increase of average molecular weights of polymers, as expected (Table 1). These polymers were soluble in medium-polarity solvents with low boiling points, such as tetrahydrofuran (THF) and chloroform, and in aprotic polar solvents, including $N, N^{\prime}$-dimethylformamide (DMF), $N, N^{\prime}$ dimethylacetamide (DMAc), and $N$-methyl-2-pyrrolidone (NMP). The solubility of all polymer samples in various solvents was sufficient for the PPH\# series to be applied as thin film materials for electro-optical devices. Additionally, the thermal properties of the PPH\# series, i.e. PPH20 to PPH100, were investigated using DSC (Table 1).

All polymers were amorphous as a single glass transition was observed in each DSC thermogram (data not shown). As the molar ratio of the phthalimide side groups in the polymers increased, the glass transition temperature $\left(T_{\mathrm{g}}\right)$ increased from $137.19{ }^{\circ} \mathrm{C}$ for PPH20 to $159.17^{\circ} \mathrm{C}$ for PPH100. In general, the $T_{\mathrm{g}}$ of polymers depends on properties such as bulkiness flexibility, and polarity of their side groups. It has been reported that the $T_{\mathrm{g}}$ of polymers can be increased with increasing side group polarity and increased or decreased with increasing bulkiness of the polymer side group. ${ }^{23}$ For instance, the $T_{\mathrm{g}}$ of poly(vinylnaphthalene) containing, e.g. naphthalene, is higher than that of PS. Thus, the incorporation of a bulky side group can increase the intermolecular interactions in the polymer. 
Table 1 Reaction conditions and characteristic of PPHO and phthalimide modified polystyrene (PPH\#)

\begin{tabular}{|c|c|c|c|c|c|}
\hline Polymer designation & $\begin{array}{l}\text { Phthalimide potassium } \\
\text { salt (mol\% compared with PPH0) }\end{array}$ & Degree of substitution (\%) & $M_{\mathrm{n}}\left(\mathrm{g} \mathrm{mol}^{-1}\right)$ & $M_{\mathrm{w}} / M_{\mathrm{n}}$ & $T_{\mathrm{g}}\left({ }^{\circ} \mathrm{C}\right)$ \\
\hline PPH0 & - & - & 22000 & 2.32 & 103.15 \\
\hline PPH40 & 40 & 40 & 26500 & 2.54 & 144.18 \\
\hline PPH60 & 60 & 60 & 28200 & 2.61 & 150.42 \\
\hline PPH80 & 80 & 80 & 30700 & 2.30 & 154.18 \\
\hline
\end{tabular}

Similarly, the incorporation of bulky phthalimide moieties affected the intermolecular interactions of the PPH\# series, which increased the $T_{\mathrm{g} .}{ }^{23}$

\subsection{Transmittance of PS films substituted with phthalimidoyl group}

The transmittance of the polymer films to visible light is an important factor for determining the visibility of the display panel on which the films are applied. Quantitative analysis of the optical transmittance of pristine PS, PI, and PPH\# series was performed using UV-Vis spectrophotometry to confirm their suitability for film coating applications (Fig. 3). The optical transmittance values of the PPH\# and PS films coated onto the glass substrates ranged from 91 to $93 \%$ at $550 \mathrm{~nm}$, which is higher than that $(80.5 \%)$ of the commonly used PI film. The PPH\# film did not exhibit an intrinsically yellowish coloration, which was ascribed to diimide fragment conjugation when used as a LC alignment layer. The optical transmittance of all films in the visible light region was sufficient for use as an optical material in electro-optical displays.

\subsection{LC alignment of the LC cells fabricated with PS substituted with phthalimidoyl group}

Fig. 4 shows the photographic and orthoscopic polarized optical microscopy (POM) images of the LC cells fabricated with PPH100 films on glass substrates using 0.02, 0.04, 0.06, 0.08, 0.1, and $1.0 \mathrm{wt} \% \mathrm{PPH} 100$. Random planar alignment was

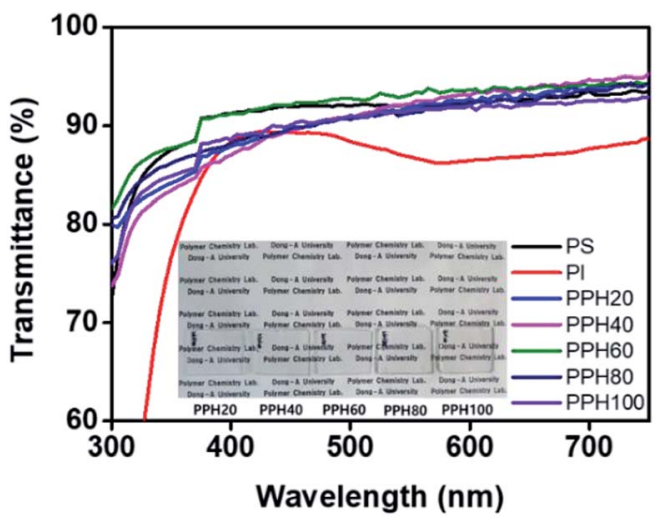

Fig. 3 UV-Vis transmittance spectra of polystyrene, polyimide, and phthalimide modified polystyrene ( $\mathrm{PPH}$ ) alignment layers on glass substrate. observed in the samples prepared with $\leq 0.08 \mathrm{wt} \%$ PPH100. At high concentrations of PPH100 ( $\geq 0.1 \mathrm{wt} \%$ ), homogeneous planar alignments were observed in the large photographic area. For LC cell prepared from PPH100 films coated with the $0.1 \mathrm{wt} \%$ solution, a LC alignment defect in the narrow area was confirmed from the photographic and orthoscopic images, but the LC cell fabricated with 1 wt\% PPH100 solution did not show the same defect. Therefore, we selected $1 \mathrm{wt} \%$ solution for fabrication of the LC cells using $\mathrm{PPH} \#$ films.

Fig. 5 shows photographs of the LC cells fabricated using PS and the PPH\# polymer series $\#=20-100)$. The LC cells composed of PS films were initially well-oriented. However, after several days, the LC alignment was disordered to adopt a random planar alignment. On the other hand, LC cells fabricated with $\mathrm{PPH}$ \# films containing phthalimide moiety contents of $>20 \mathrm{~mol} \%$ (PPH20, PPH40, PPH60, PPH80, and PPH100) showed clearly homogeneous planar LC alignment behavior over the entire photographed area, unlike the device prepared with PS films. To investigate the alignment of LC molecules systematically, POM images were obtained in orthoscopic mode and shown in Fig. 6. As mentioned above, the LC alignment of the LC cell fabricated with PS films converted to a random planar alignment after several days (Fig. 6(a)). Additionally, consistent LC alignment behavior of the LC cells fabricated with $\mathrm{PPH}$ \# films was verified using the orthoscopic POM images in Fig. 6(b)-(f). The aligning ability of the LC cells fabricated with rubbed PPH\# films showed similar LC alignment behavior as those of the LC cells composed of PI films. This was determined by observing photographs with the naked eye under crossed polarizer. The LC cells fabricated with $\mathrm{PPH \#}$ films were superior to those composed of PS films regarding to long-term stability.

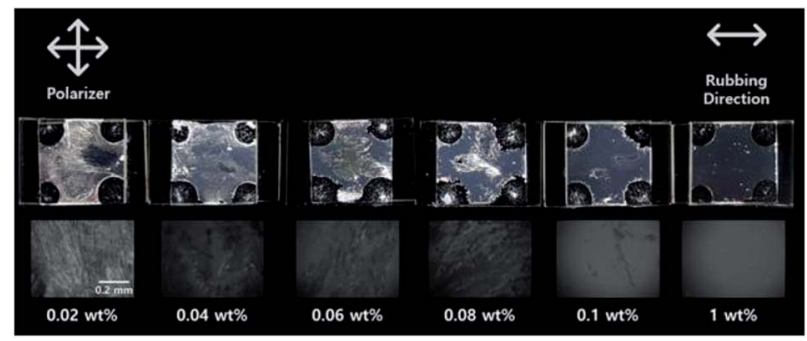

Fig. 4 Photograph images and orthoscopic POM images of the LC cells fabricated with $\mathrm{PPH} 100$ films under the weight ratio of the $\mathrm{PPH} 100$ solution. 


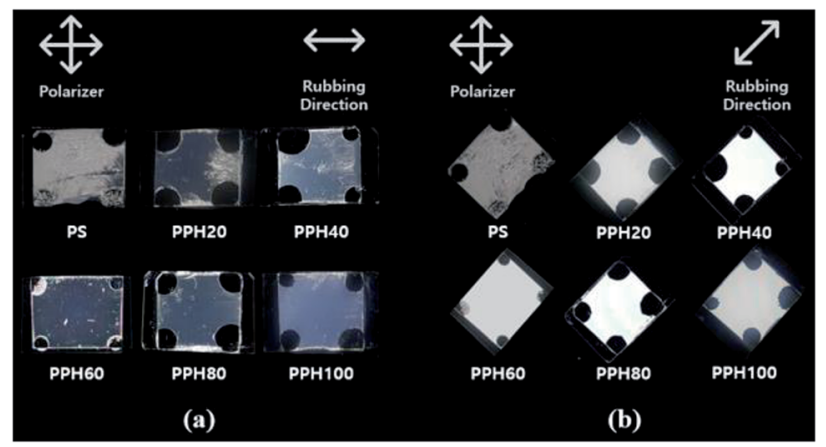

Fig. 5 Photograph images of the LC cells fabricated with polystyrene and phthalimide modified polystyrene (PPH\#) (\# = 20,40,60, 80, and 100) films viewed under crossed polarizers by rotating LC cells in (a) zero degrees and (b) 45 degrees.

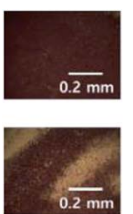

(a)

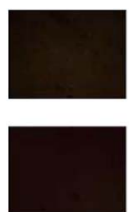

(b)

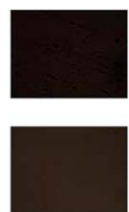

(c)

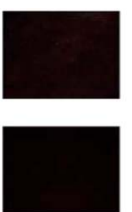

(d)

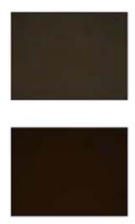

(e)
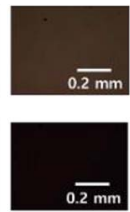

(f)

Fig. 6 Orthoscopic POM images of the initial (top) and several-dayold (bottom) LC cells made from (a) polystyrene, (b) PPH2O, (c) PPH40, (d) PPH60, (e) PPH80, and (f) PPH100.

\subsection{Angular dependence of the LC orientation in LC cells fabricated with PS substituted with phthalimidoyl group}

The angular dependence of the LC alignment direction was investigated by IR absorbance to systematically confirm the alignment of the LC molecules in the LC cell. The LC alignment directions of the antiparallel LC cells made from the rubbed PS and $\mathrm{PPH} \#$ polymer films coated on the Si-wafer were determined by the angular dependence of the absorbance of nitrile $(\mathrm{C} \equiv \mathrm{N})$ in $5 \mathrm{CB}$ at $2226 \mathrm{~cm}^{-1}$ as a function of the dichroic angle $\left(0\right.$ and $\left.90^{\circ}\right)$ of the polarizer using polarized FT-IR measurements in Fig. 7. From Fig. 7(a), it is clear that IR adsorption intensity of the nitrile $(\mathrm{C} \equiv \mathrm{N})$ group in $5 \mathrm{CB}$ at $90^{\circ}$ is greater than that obtained at $0^{\circ}$. This indicates that the LC molecules on the PS film were aligned
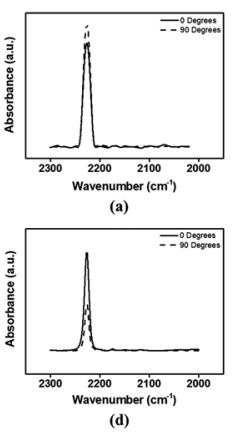
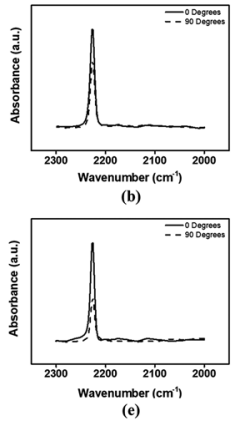
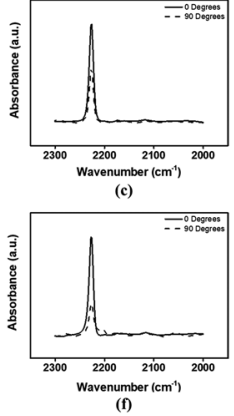

Fig. 7 FT-IR dichroic spectra of LC silicon wafer cells fabricated with rubbed (a) polystyrene, (b) PPH20, (c) PPH40, (d) PPH60, (e) PPH80, and $(\mathrm{f}) \mathrm{PPH} 100$ films detecting nitrile bond $(\mathrm{C} \equiv \mathrm{N})$ of $5 \mathrm{CB}$.

more dominantly in the perpendicular direction with respect to the rubbing direction than that in parallel direction. However, from Fig. 7(b)-(f), the IR absorbance intensity of the nitrile $(\mathrm{C} \equiv \mathrm{N})$ group at $0^{\circ}$ is greater than that at $90^{\circ}$. This indicates that the $5 \mathrm{CB}$ molecules on the PPH\# films were aligned more dominantly in a parallel direction, opposite to that of Fig. 7(a). Interestingly, according to Figs. 7(b)-(f), as the degree of substitution of phthalimidoyl groups in PPH\# increases (from \# = 20 to \#=100), peak intensity as 90 degrees has a tendency to be decreased gradually. This means that as the number of phthalimidoyl moieties increases, the alignment direction of LC molecules is preponderantly oriented parallel with respect to the rubbing direction. To elucidate the LC alignment direction accurately, the angular dependence from 0 to $360^{\circ}$ of the FT-IR absorbance of the antiparallel LC cells fabricated with rubbed PS and PPH\# films coated onto Si-wafers was determined from the FT-IR polar diagrams (Fig. 8). The antiparallel LC cells composed of rubbed PS films showed maximum intensities along the $90^{\circ} \leftrightarrow 270^{\circ}$ direction by monitoring the polar diagram of the intensity of the nitrile $(\mathrm{C} \equiv \mathrm{N})$ group stretching band in the $5 \mathrm{CB}$ molecules as a function of the rotational angle of the polarizer. The results indicated that the LC molecules on the PS films were aligned perpendicularly with respect to the rubbing direction (Fig. 8(a)), which is consistent with the literature. ${ }^{24}$ For the $\mathrm{PPH} \#$ series, the maximum absorbance was observed along the $0^{\circ} \leftrightarrow 180^{\circ}$ direction after the rubbing process, indicating that the LC cells fabricated with all PPH\# films adopted an LC alignment parallel with respect to the rubbing direction. Therefore, we concluded that the LC alignment direction can be switched from perpendicular to parallel by introducing a phthalimide group in the PS side chain.

The LC alignment direction depends on the molecular orientation and surface morphology of the polymer layer after rubbing treatment. We believe that the molecular orientation of polymeric alignment layer after rubbing process can be switched preferentially. In this case, aliphatic backbone and aliphatic/ aromatic side chain can be reoriented in parallel and/or perpendicular with respect to the rubbing direction. Based on this view, anisotropic interaction between LC molecules and preferred-oriented polymer side chain, $\pi-\pi$ interaction with each phenyl groups in mesogenic and phthalimidoyl group, can be
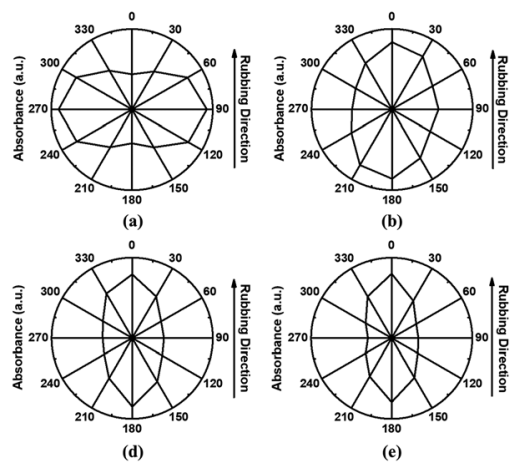

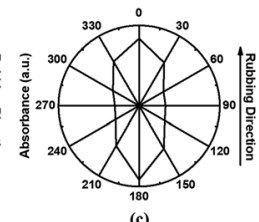

(c)

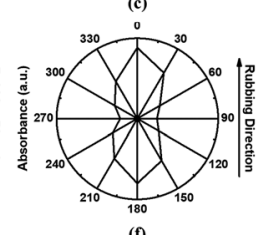

Fig. 8 Polar diagram of specific vibrational IR peaks of nitrile $(\mathrm{C} \equiv \mathrm{N})$ of 5 CB in LC silicon wafer cells fabricated with rubbed (a) polystyrene, (b) $\mathrm{PPH} 20$, (c) $\mathrm{PPH} 40$, (d) $\mathrm{PPH} 60$, (e) $\mathrm{PPH} 80$, and (f) $\mathrm{PPH} 100$ films measured as a function of rotational angle of polarizer. 
formed. Changes in the LC molecular orientation on the PPH100 film caused by rubbing is expected to be observed via AFM.

\subsection{Surface morphology of the PS films substituted with phthalimidoyl groups}

The surface morphology of the polymer films was examined using AFM (Fig. 9). From the AFM 2D and 3D images before the rubbing process (Fig. 9(a) and (b), respectively), the surface morphology of the non-rubbed PPH100 film with a $R_{\mathrm{a}}$ (average roughness) of approximately $2.07 \mathrm{~nm}$ showed submicro- and/or subnano-scale polka-dot-like structures. However, in the 2D and 3D AFM images obtained after the rubbing process (Fig. 9(c) and (d)), the rubbed PPH100 film exhibited groove-like structures parallel with respect to the rubbing direction. It was difficult to observe the submicro- and/or subnano-scale groovelike structures on the rubbed PPH100 film due to the high roughness of approximately $2.51 \mathrm{~nm}$. We attempted to observe the surface morphology change of the PPH100 film fabricated under different coating conditions. However, we could not observe distinct changes in $R_{\mathrm{a}}$ of the PPH100 films according to spin-coating speed and time (data not shown). Therefore, the $\mathrm{PPH}$ \# film was fabricated using a $1 \mathrm{wt} \%$ solution by spin-coating (2000 rpm, $60 \mathrm{~s}$ ). We assumed that the rough surface of the PPH100 film makes it difficult to distinguish differences in the groove-like structure. Generally, LC molecules are oriented parallel with respect to the rubbing direction, ${ }^{\mathbf{1 4}}$ which may lead to similarities in the LC alignment properties between the PPH100 and PI films. ${ }^{25-27}$ Therefore, we can conclude that the groove-like structure of the polymer surface is a core element required for obtaining a clear and planar LC alignment in terms of morphological properties.

\subsection{Ultraviolet stability of the PS substituted with phthalimidoyl groups}

The credibility of the LC cells composed of the polymer films was established via LC aligning stability evaluation under harsh conditions that included UV irradiation. The UV stabilities of
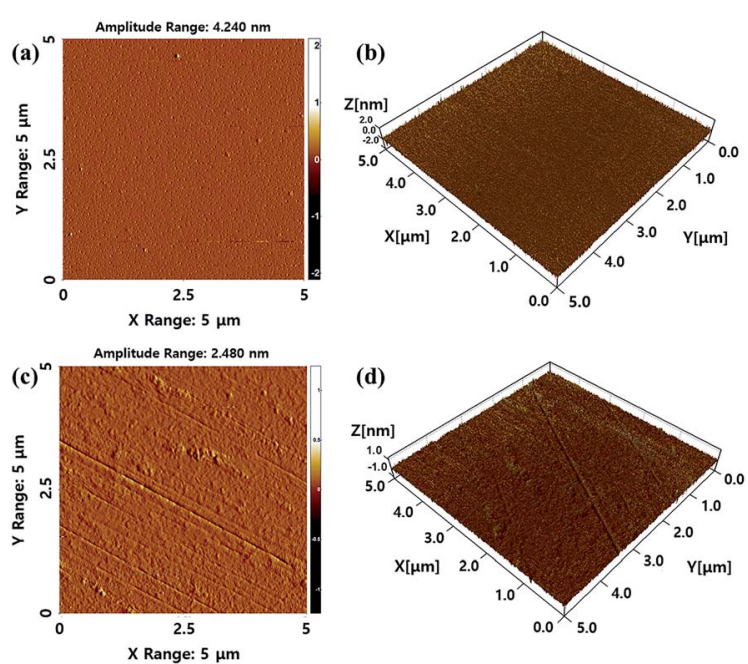

Fig. 9 AFM images of the unrubbed PPH100 film ((a) 2D and (b) 3D) and rubbed PPH100 film ((c) 2D and (d) 3D) on mica sheet.
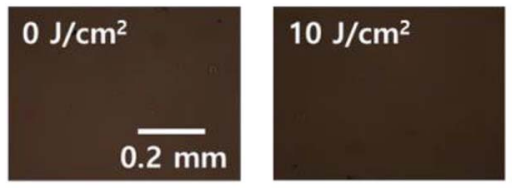

(a)

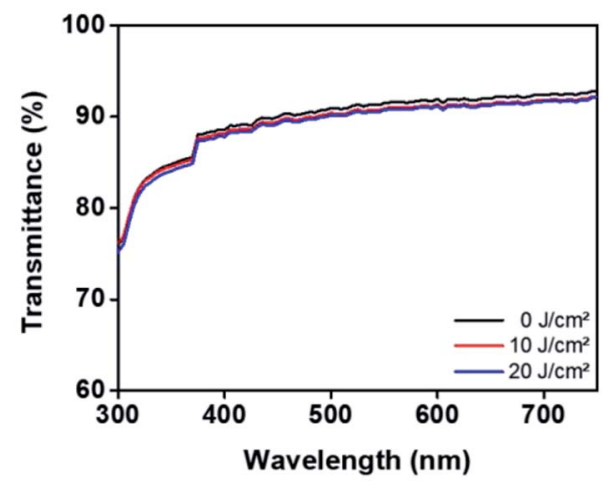

(b)
Fig. 10 (a) Orthoscopic POM images of the LC cells made with $\mathrm{PPH} 100$ films and (b) UV-Vis transmittance spectra of PPH100 films, before $\left(0 \mathrm{~J} \mathrm{~cm}^{-2}\right)$ and after UV treatment at 10 and $20 \mathrm{~J} \mathrm{~cm}^{-2}$, respectively.

the LC cells composed of PPH\# polymers from PPH20 to PPH100 were estimated from orthoscopic POM images obtained after UV irradiation at 10 and $20 \mathrm{~J} \mathrm{~cm}^{-2}$. As shown in Fig. 10(a), distinguishable differences in the parallel LC alignment on PPH100 film were not observed in the orthoscopic POM images, indicating that the parallel LC aligning ability of the PPH100 LC cell was maintained even at high UV energy. The parallel LC aligning ability of the copolymer (PPH20, PPH40, PPH60, and PPH80) LC cells was maintained at high UV energy (20 $\mathrm{J} \mathrm{cm}^{-2}$; data not shown). Further, to confirm UV stability of polymeric alignment layer (PPH100), the polymer films were irradiated with UV ray under the same condition, and then their transmittance was examined. According to Fig. 10(b), it was confirmed that transmittance of PPH100 film before UV irradiation and those of the film after irradiation in 10 and $20 \mathrm{~J}$ $\mathrm{cm}^{-2}$ were almost corresponded, respectively. We concluded that PPH100 has excellent stability against UV ray. Therefore, based on these results, the $\mathrm{PPH} \#$ series can enhance the UV stability of the corresponding LC cells and is a candidate for use in next generation LC alignment layers.

\section{Conclusions}

A series of PS substituted with phthalimide ( $\mathrm{PPH}$ ) containing the isoindoline-1,3-dione moiety, a representative moiety in heterocyclic PI, were synthesized to investigate the LC alignment properties of PPH\# films. PPH100 polymer films exhibited good optical transparency in the visible region $(400-700 \mathrm{~nm})$. The transmittance value (91.6\%) of the PPH100 film on the glass substrate at $550 \mathrm{~nm}$ was higher than that of the PI film (80.5\%), the most commonly used polymer for LC alignment layers. The LC cell fabricated with PS was oriented perpendicular with 
respect to the rubbing direction and the long-term and UV stabilities of the LC cells were remarkably low. On the other hand, the LC alignments of the LC cells composed of PPH\#s were oriented parallel with respect to the rubbing direction. For instance, the LC cells incorporating films fabricated with polystyrenes with $>20 \mathrm{~mol} \%$ phthalimide showed a homogeneously planar LC alignment parallel with respect to the rubbing direction. These results suggest that the orientation of the LC molecules changed from perpendicular to parallel with respect to the rubbing direction by introducing phthalimide groups into the PS system. Moreover, the long-term and UV stabilities of the LC cells were enhanced compared to those of the LC cells fabricated with PS films. This study provides support for the design of parallel LC alignment layers using phthalimide groups in polymer side chains.

\section{Conflicts of interest}

There are no conflicts to declare.

\section{Acknowledgements}

Financial supports by the Dong-A University Research Fund are gratefully acknowledged.

\section{Notes and references}

1 G. H. Brown, Advances in liquid crystals, Academic Press, New York, vol. 2, 1976.

2 G. Vertogen and W. H. de Jeu, Thermotropic liquid crystal, fundamentals, Springer-Verlag, Berlin, 2012.

3 D. H. Chung, Y. Takahashi, K. Ishikawa, H. Takezoe, B. Park, Y. Jung, H. K. Hwang, S. Lee, K. J. Han and S. H. Jang, Jpn. J. Appl. Phys., 2000, 39, 185.

4 S. G. Kim, S. M. Kim, Y. S. Kim, H. K. Lee and S. H. Lee, Appl. Phys. Lett., 2007, 90, 261910.

5 M. Lee, J. W. Bae, A. Kim, H. S. Yun and K. Song, Polymer (Korea), 2015, 39, 174.

6 Y. Kim, M. Lee, H. S. Wang and K. Song, Opt. Mater. Express, 2017, 7, 4225.
7 A. Bacher, P. G. Bentley, D. D. C. Bradley, L. K. Douglas, P. A. Glarvey, M. Grell, K. S. Whitehead and M. L. Turner, J. Mater. Chem., 1999, 9, 2985.

8 P. G. de Gennes, C. R. Acad. Sci., Ser. IIb: Mec., Phys., Chim., Astron., 1997, 324, 343.

9 X. Wang, J. Engel and C. Liu, J. Micromech. Microeng., 2003, 13, 628.

10 S. J. Woltman, G. D. Jay and G. P. Crawford, Nat. Mater., 2007, 6, 929.

11 P. Fischer, A. Laschewsky, E. Wischerhoff, X. Arys, A. Jonas and R. Legras, Macromol. Symp., 1999, 137, 1.

12 G. Rabiloud, High-performance polymers, Edition Technip, Paris, 2000.

$13 \mathrm{~W}$. Zheng, Surface wetting characteristics of rubbed polyimide thin films, Polym. Thin Films, InTech, London, ch. $10,2010$.

14 D. W. Berreman, Phys. Rev. Lett., 1972, 28, 1683.

15 S. Ando, T. Matsuura and S. Sasaki, Polym. J., 1997, 29, 69.

16 H. S. Nalwa, Supramolecular photosensitive and electroactive materials, Academic Press, New York, 2001.

17 S. Ishihara, H. Wakemoto, K. Nakazima and Y. Mastuo, Liq. Cryst., 1989, 4, 669.

18 D.-S. Seo, K.-I. Muroi, T.-R. Isogami, H. Matsuda and S. Kobayashi, Jpn. J. Appl. Phys., 1992, 31, 2165.

19 M. Oh-e, S.-C. Hong and Y. R. Shen, Appl. Phys. Lett., 2002, 80, 784.

20 S. G. Hahm, T. J. Lee, S. W. Lee, J. Yoon and M. Ree, Mater. Sci. Eng., B, 2006, 132, 54.

21 S. W. Lee, J. Yoon, H. C. Lee, B. Lee, T. Chang and M. Ree, Macromolecules, 2003, 36, 9905.

22 H. Kang and D. Kang, Mol. Cryst. Liq. Cryst., 2015, 623, 45.

23 U. W. Gedde, Polymer physics, Springer Science+Business Media, Berlin, 1999.

24 K. Nakajima, H. Wakemoto, S. Sato, F. Yokotani, S. Ishihara and Y. Matsuo, Mol. Cryst. Liq. Cryst., 1990, 180B, 223.

25 J. M. Geary, J. W. Goodby, A. R. Kmetz and J. S. Patel, J. Appl. Phys., 1987, 62, 4100.

26 N. A. J. van Aerle and J. W. Tol, Macromolecules, 1994, 27, 6520.

27 S. I. Kim, S. M. Pyo, M. Ree, M. Park and Y. Kim, Mol. Cryst. Liq. Cryst., 1998, 316, 209. 